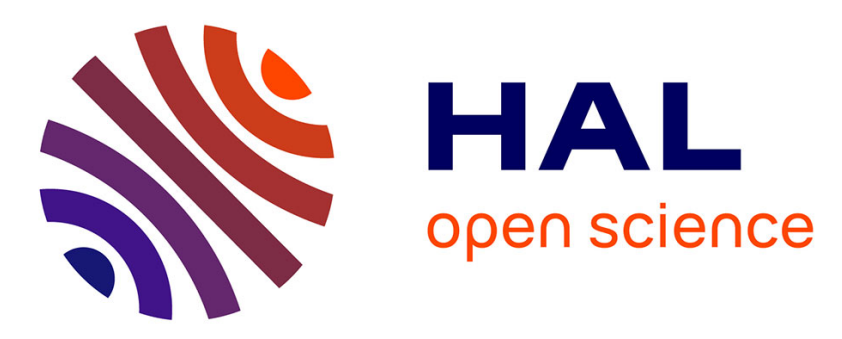

\title{
Role of pedunculopontine cholinergic neurons in the vulnerability of nigral dopaminergic neurons in Parkinson's disease
}

\author{
Manale Bensaid, Patrick P. Michel, Stewart D. Clark, Etienne C. Hirsch, \\ Chantal François
}

\section{To cite this version:}

Manale Bensaid, Patrick P. Michel, Stewart D. Clark, Etienne C. Hirsch, Chantal François. Role of pedunculopontine cholinergic neurons in the vulnerability of nigral dopaminergic neurons in Parkinson's disease. Experimental Neurology, 2016, 275 (3), pp.209-219. 10.1016/j.expneurol.2015.11.004 . hal-01229085

\section{HAL Id: hal-01229085 \\ https://hal.sorbonne-universite.fr/hal-01229085}

Submitted on 16 Nov 2015

HAL is a multi-disciplinary open access archive for the deposit and dissemination of scientific research documents, whether they are published or not. The documents may come from teaching and research institutions in France or abroad, or from public or private research centers.
L'archive ouverte pluridisciplinaire HAL, est destinée au dépôt et à la diffusion de documents scientifiques de niveau recherche, publiés ou non, émanant des établissements d'enseignement et de recherche français ou étrangers, des laboratoires publics ou privés. 
Role of pedunculopontine cholinergic neurons in the vulnerability of nigral dopaminergic neurons in Parkinson's disease

Manale Bensaid ${ }^{\mathrm{a}}$, Patrick P. Michel ${ }^{\mathrm{a}}$, Stewart D. Clark ${ }^{\mathrm{b}}$, Etienne C. Hirsch ${ }^{\mathrm{a}}$, Chantal François $^{\mathrm{a}}$

${ }^{a}$ Sorbonne Universités, UPMC Univ Paris 06, INSERM, CNRS, UM75, U1127, UMR 7225, ICM, F-75013 Paris, France

${ }^{b}$ Department of Pharmacology and Toxicology, University at Buffalo, Buffalo, New York 14214

Corresponding author: Chantal François, Sorbonne Universités, UPMC Univ Paris 06, INSERM, CNRS, UM75, U1127, UMR 7225, ICM, F-75013 Paris, France. Phone number: +33 1572745 40; E-mail: Chantal.francois@upmc.fr

Number of Figures: 7

Number of Tables: 1

Number of pages: 37 


\begin{abstract}
Pedunculopontine nucleus (PPN) cholinergic neurons, which exert excitatory nicotinic control over substantia nigra dopaminergic neurons, degenerate in Parkinson's disease (PD). This finding and other studies showing that nicotine, the preferential agonist of nicotinic acetylcholine receptors, is neuroprotective in experimental models of PD suggest that a deficit in PPN excitatory cholinergic inputs might contribute to the death of nigral dopaminergic neurons in PD. To explore this possibility, we used lesion paradigms of dopaminergic and/or cholinergic systems in rats and monkeys. Consistent with our hypothesis, we observed that stereotaxic lesioning of PPN cholinergic neurons with diphtheria toxin coupled to urotensin II resulted in a significant loss of nigral dopaminergic neurons in rats and induced morphological changes in these neurons in macaques. Unexpectedly, a lesion of dopaminergic neurons induced by unilateral striatal injection of 6-hydroxydopamine (6-OHDA) in rats, or by repeated systemic injections of 1-methyl-4-phenyl-1,2,3,6-tetrahydropyridine (MPTP) in macaques, led to a $29 \%$ and $7 \%$ loss of PPN cholinergic neurons, respectively. Lastly, when the PPN cholinergic lesion was performed in rats in which the dopaminergic lesion induced by 6-OHDA was in progress, loss of cholinergic neurons was more drastic than when each neurotransmitter system was lesioned separately. Thus, our results suggest that strong PPN cholinergic and dopaminergic interactions may be an important mechanism in the pathophysiology of PD.
\end{abstract}


Key words: pedunculopontine nucleus, cholinergic, Parkinson's disease, dopamine, 6-OHDA, MPTP, diphtheria toxin-urotensin II, substantia nigra

Abbreviations: ChAT, choline acetyl transferase; DA, dopaminergic; Dtx-UII, diphtheria toxin coupled to urotensin II; Iba-1, ionized calcium-binding adaptor molecule 1; MPTP, 1 methyl-4-phenyl-1,2,3,6-tetrahydropyridine; NADPH, nicotinamide adenine dinucleotide phosphate diaphorase; NeuN, neuronal nuclear protein; PD, Parkinson's disease; PPN, pedunculopontine nucleus; SNc, substantia nigra pars compacta; $\mathrm{TH}$, tyrosine hydroxylase; 6OHDA, 6-hydroxydopamine. 


\section{Introduction}

Parkinson's disease (PD) results primarily from a loss of nigrostriatal dopaminergic (DA) neurons, but non-DA neurons also degenerate in the course of the disease. In particular, cholinergic neurons of the pedunculopontine nucleus (PPN) are also affected (Hirsch et al., 1987; Jellinger, 1988), and the extent of loss of these neurons correlates with the level of DA denervation (Zweig et al., 1989; Karachi et al., 2010). A number of arguments support the hypothesis that a deficit in cholinergic inputs may contribute to the progression of DA neuronal death in PD: 1) The PPN sends massive excitatory inputs onto DA neurons of the substantia nigra pars compacta (SNc) (Lavoie and Parent, 1994) and the nicotinic cholinergic component of this innervation is involved in the control of DA release at the striatal level (Blaha et al., 1996). This suggests that key interactions exist between PPN cholinergic neurons and nigral DA neurons. 2) Nicotinic receptors are present on nigral DA neurons (Nastuk and Graybiel, 1991; Sorenson et al., 1998), and nicotine, the preferential agonist of nicotinic acetylcholine receptors, is neuroprotective in several experimental models of PD (Costa et al., 2001; Parain et al., 2003; Quik et al., 2012). 3) Moreover, epidemiological studies have identified tobacco smoking as the most significant protective factor against the risk of developing PD (Hernan et al., 2002; Thacker et al., 2007). Taken together, these data indicate that PPN excitatory cholinergic inputs might be important for keeping alive vulnerable nigral DA neurons, possibly because these neurons have specific needs for stimulation in order to survive (Salthun-Lassalle et al., 2004; Michel et al., 2013). This means that the cholinergic PPN lesion observed in PD may accelerate the lesioning of DA neurons in this pathology. Previous studies also demonstrated that extensive lesioning or reversible inactivation of the PPN leads to a parkinsonian syndrome in macaques (Kojima et al., 1997; Aziz et al., 1998; MunroDavies et al., 1999; Matsumura and Kojima, 2001). Besides, an extensive excitotoxic lesion of the PPN was reported to induce DA neuronal loss in the SNc 
of rats (McGeer and McGeer, 1984; Gonzalez-Hernandez et al., 1997), and unilateral PPN lesion using N-methyl-D-aspartate (NMDA) induced oxidative stress events in the SNc and in the striatum (Jimenez-Martin et al., 2015). Yet, in these different paradigms, the lesion or inactivation of the PPN affected both cholinergic and non-cholinergic neurons. Thus, no definite conclusion can be drawn on the specific role of cholinergic neurons in the progression of DA cell loss.

Our aim was to test the hypothesis that a PPN lesion specific to cholinergic neurons leads to a DA nigral neuronal loss and that adding a PPN cholinergic lesion to a DA lesion, as observed in patients with PD, would aggravate DA neurodegeneration. We used lesion paradigms of cholinergic and/or DA systems in normal and parkinsonian rats and monkeys, and quantified the number of cholinergic neurons in the PPN and of DA neurons in the SNc. PPN lesions were performed using diphtheria toxin coupled to urotensin II (Dtx-UII), a toxin specific to cholinergic neurons (Clark et al., 2007). We used the intrastriatal 6hydroxydopamine (6-OHDA) lesion paradigm in order to obtain a progressive DA loss in the SNc starting between 6 and 9 days post-surgery (Sauer and Oertel, 1994; Blandini et al., 2007). We also studied macaques intoxicated with 1-methyl-4-phenyl-1,2,3,6tetrahydropyridine (MPTP) because they more closely reflect patients with PD.

\section{Material and methods}

\section{Animals}

All studies were carried out in accordance with the European Communities Council Directive of 2010 (2010/63/UE). The authorization for conducting our experiments was approved by the Ethics Committee in Animal Experiment Charles Darwin (agreement no. Ce5/2012/086). The experimental protocol was designed to minimize the number of animals 
used and to minimize their suffering. The animals were housed two per cage at $20-23^{\circ} \mathrm{C}$ with a 12:12 h dark-light cycle. Food and water were available ad libitum throughout.

\section{Experimental groups}

Thirty-five adult male Sprague-Dawley rats (250-300 g) were used in this study. The rats were divided into four groups: 1) a group with a sham lesion into the right striatum followed 1 week later by a bilateral cholinergic lesion of the PPN using the selective Dtx-UII (Clark et al., 2007) (Dtx-UII-lesioned rats, $n=11)$; 2) a group with a unilateral, partial injection of 6-OHDA into the right striatum, followed one week later by a sham lesion of the right PPN, and one week later by a sham lesion of the left PPN (6-OHDA-lesioned rats, $n=$ 13); 3) a double-lesioned group with a unilateral 6-OHDA striatal injection followed one then two weeks later, thus when DA neurons were degenerating (Sauer and Oertel, 1994; Blandini et al., 2007), by a bilateral injection of Dtx-UII into the PPN (double-lesioned rats, $n=4$ ); 4) a sham-lesioned group that received an equal volume of vehicle solution in both the striatum and the PPN (sham-lesioned rats, $n=5$ ) and control rats that received no injection at all (control rats, $n=2$ ).

We also used brain sections obtained from 16 male adult macaques (Macaca fascicularis) previously used in two other studies (Karachi et al., 2010; Grabli et al., 2013): six macaques had been intoxicated with MPTP, five had received a cholinergic lesion of the PPN using Dtx-UII, and five others were considered as a control group.

\section{DA and cholinergic lesions in rats}

Surgical procedures were performed under general anesthesia with ketamine $(50 \mathrm{mg} / \mathrm{kg}$, Imalgene 500; Merial, Lyon, France) and xylazine (10 mg/kg, Rompun 2\%; Bayer, 
Leverkusen, Germany) administered intramuscularly. Rats received pre- and post-operative analgesia with carprofen (rimadyl $5 \mathrm{mg} / \mathrm{kg}, \mathrm{sc}$ ).

The rat was placed in a stereotactic frame (David Kopf Instruments) on an electronically controlled isothermic heating pad. During surgery, anesthesia was then induced with a mixture of isoflurane (3\% induction, $2 \%$ maintenance dose) and oxygen $(1.5 \mathrm{~L} / \mathrm{min})$. Injection sites were determined according to the atlas of Paxinos and Watson (2005) and all injections were slowly infused $(0.2 \mu \mathrm{l} / \mathrm{min})$. All animals except the 2 controls underwent 3 surgeries in order to lesion the striatum unilaterally, followed 1 week later by a bilateral cholinergic lesion of the PPN. To avoid surgical risk with brainstem surgery, the neurotoxin was first injected unilaterally into the right PPN, then, one week later, a contralateral injection was performed using the same procedure. We chose to lesion the striatum unilaterally to reduce the risk of animal death due to the 3 successive surgeries and because bilateral nigrostriatal lesion carries an additional risk of death.

Bilateral lesions of the PPN were made by giving two infusions of $1 \mu \mathrm{L}$ of Dtx-UII (5\%) at bregma $-7.6 \mathrm{~mm}$, midline $\pm 1.8 \mathrm{~mm}$, dura $-7.3 \mathrm{~mm}$, and bregma $-8.4 \mathrm{~mm}$, midline \pm 1.8 $\mathrm{mm}$, dura $-6.9 \mathrm{~mm}$. Lesions of the right striatum were made by giving two infusions of 6OHDA ( $2 \times 2 \mu \mathrm{L}, 4 \mu \mathrm{g} / \mu \mathrm{l}$ in a $0.01 \%$ ascorbic acid solution; Sigma) at the following stereotaxic coordinates: bregma $+0.5 \mathrm{~mm}$, midline $+2.5 \mathrm{~mm}$, dura $-5 \mathrm{~mm}$; and bregma -0.5 $\mathrm{mm}$, midline $+4 \mathrm{~mm}$, dura $-5 \mathrm{~mm}$. The animals were kept under a heating lamp until they had fully recovered from anesthesia, after which they were returned to their cage and housed individually. 
The MPTP regimen consisted of intramuscular injections $(0.2-0.4 \mathrm{mg} / \mathrm{kg})$ at an interval of 3 to 7 days until the emergence of all parkinsonian symptoms. All six MPTP-treated macaques developed severe motor symptoms and were euthanized after a survival time of 2 to 4 months. Dtx-UII $(10 \mu 1,20 \%)$ was injected stereotactically into the PPN, first unilaterally, and then contralaterally 2-4 weeks later, as previously described (Karachi et al., 2010). Monkeys were killed 2 months after the last lesion. After transcardial perfusion with $4 \%$ paraformaldehyde, brains were removed, frozen and cut transversely into 50- $\mu$ m-thick sections.

\section{Tissue collection}

All 32 rats were killed 7 weeks after the first striatal saline or 6-OHDA injection, except for five 6-OHDA-lesioned rats that were killed 15 weeks after the striatal lesion (6-OHDAlesioned-15w rats). Each rat was killed by a lethal intraperitoneal injection of sodium pentobarbital and perfused through the heart with $\sim 100 \mathrm{ml}$ of $0.1 \mathrm{M}$ phosphate-buffered saline (PBS, pH 7.4), followed by $-300 \mathrm{ml} 4 \%$ paraformaldehyde in $0.1 \mathrm{M}$ PBS (pH 7.4). Brains were removed and placed into $4 \%$ paraformaldehyde overnight for post-fixation prior to transfer to PBS-20\% sucrose and frozen. Transverse $40-\mu \mathrm{m}$-thick sections were cut on a freezing microtome.

\section{Peroxidase immunohistochemistry}

Parallel series of regularly interspaced $(400 \mu \mathrm{m}$ apart for rats and $500 \mu \mathrm{m}$ apart for macaques) fresh-frozen sections of brains were immunohistochemically processed for tyrosine hydroxylase (TH) using a mouse monoclonal anti-TH (1:500; Immunostar, Hudson, WI), choline acetyltransferase (ChAT) using a goat polyclonal anti-ChAT (1:500, Chemicon, Merck Millipore), a neuron-specific nuclear protein using mouse monoclonal anti-neuronal 
nuclear protein (NeuN) (1/500; Chemicon, Merck Millipore), or a rabbit polyclonal antibody against ionized calcium-binding adaptor molecule 1 (Iba-1) (1:500; WAKO, Richmond, VA). They were then incubated with secondary antibodies from appropriate species and the signal was amplified using Vectastain ELITE ABC reagents (Vector Laboratories, Inc., Burlingame, CA). The antibodies were then visualized by peroxidase histochemistry with diaminobenzidine (Sigma) as substrate. Sections were counterstained with Nissl stain, cresyl violet or neutral red. Some sections were also processed for Fluoro-Jade B staining using the Fluoro-Jade B Ready-to-dilute staining kit (Chemicon International/Millipore, Saint-Quentinen-Yvelines, France), to detect degenerating neurons according to the procedure described elsewhere (Schmued and Hopkins, 2000).

\section{Double fluorescent immunochemistry}

Other series of regularly interspaced sections were incubated with rabbit anti-Iba-1 (1:500; WAKO, Richmond, VA), and with goat polyclonal anti-ChAT (1:500). Sections were then incubated with secondary fluorescent antibodies from the appropriate species (all from Life Technologies), and nuclei were stained with 4',6'-diamino-2-phenylindole dihydrochloride (DAPI).

Another series of regularly interspaced sections was processed for nicotinamide adenine dinucleotide phosphate diaphorase (NADPH) histochemistry, as previously described (Hirsch et al., 1987). NADPH was used to label cholinergic PPN neurons; virtually every cholinergic neuron within the PPN contains NADPH-diaphorase activity in rats and monkeys (Geula et al., 1993).

\section{Quantification}


TH-positive $(\mathrm{TH}+)$ cell bodies and fibers and NADPH diaphorase-positive (NADPH+) cell bodies were quantified in all animals. The investigator performing the quantification was blinded to the treatment during the analysis. The sections were matched anatomically in each of the brains, while verifying that the cross-sections of the SNc and of the PPN were similar in all individuals.

1) Optical densities of the $\mathrm{TH}+$ fibers in the striatum, represented by gray means, were measured using image analysis software (MCID Image Analysis Software Solutions for Life Sciences, Interfocus Imaging Ltd., Linton, UK). In rats, the entire dorsal striatum, excluding the ventrally located nucleus accumbens, was outlined and measured in 5 to 9 striatal sections per animal (corresponding to +1.2 to $-0.8 \mathrm{~mm}$ relative to bregma, Paxinos and Watson, 1998). In macaques, the putamen caudal to the anterior commissure was outlined and measured in 2 different sections separated by $2 \mathrm{~mm}$. A background measurement (taken in the corpus callosum) was subtracted from each measurement in the striatum, and the mean was calculated. The data from the lesioned striatum were presented as a percentage (mean \pm SEM) of the values from the striatum of sham-lesioned rats, or from the putamen of control macaques.

2) Estimation of the total number of $\mathrm{TH}+$ cell bodies in the $\mathrm{SNc}$ was performed using an unbiased stereology method with a computer-based system (Mercator, ExploraNova, La Rochelle, France) as described previously (Karachi et al., 2010). The method consists in delineating the $\mathrm{SNc}$ in regularly spaced sections (every $400 \mu \mathrm{m}$ in rats and $500 \mu \mathrm{m}$ in macaques) covering the antero-posterior extent of the structure of all animals. TH+ cell bodies were counted and sampled according to the so-called fractionator principles, that is, a combination of the optical dissector, a three-dimensional probe used for counting, and fractionator sampling, a scheme involving the probing of a known fraction of the tissue (West et al., 1991). Optical dissectors were distributed using a systematic sampling scheme. The 
height of the dissector was $22 \mu \mathrm{m}$ in macaques and $18 \mu \mathrm{m}$ in rats. Dissectors ( $60 \mu \mathrm{m}$ long, 60 $\mu \mathrm{m}$ wide) were separated from each other by $75 \mu \mathrm{m} \times 75 \mu \mathrm{m}$. To be counted, a neuron had to come into focus within the optical dissector, and had to be entirely within the surface area of the dissector or had to cross the dissector border. Only two consecutive borders on four of the dissectors were considered.

3) The total number of Iba-1+ microglia cells in the SNc was counted on Iba1-stained sections in sham-lesioned and Dtx-UII-lesioned rats using the same fractionator and optical dissector method as described above. The method consists in delineating the SNc in 3 spaced sections (from -4.80 to $-6.04 \mathrm{~mm}$ relative to bregma, Paxinos and Watson, 1998), the 3 section levels being identical between all animals. The number of Iba+ cells was expressed as cells per $\mathrm{mm}^{2}$.

4) NADPH+ cell bodies were quantified in regularly spaced sections of the PPN (every $400 \mu \mathrm{m}$ in rats and $500 \mu \mathrm{m}$ in macaques) covering the antero-posterior extent of the structure of all animals using the same image analysis system (Mercator, ExploraNova). The cholinergic neurons serve to delineate PPN boundaries. They correspond to the Ch5 cholinergic population according to the nomenclature proposed by Mesulam et al. (1983). They were distinguished from the Ch6 cholinergic neurons of the laterodorsal tegmental nucleus lying within the periaqueductal gray matter, dorsal and caudal to the PPN (Mesulam et al., 1983). The total number of NADPH+ cell bodies present in the PPN sections was estimated by plotting all cells per section. The Abercrombie correction factor $(\mathrm{N}=\mathrm{n} \times \mathrm{T} / \mathrm{T}+$ D), where $\mathrm{N}$ is the actual number of cells, $n$ the number of counted cells, $T$ the section thickness $(50 \mu \mathrm{m}$ in macaques and $40 \mu \mathrm{m}$ in rats), and $D$ the average diameter of nuclei (16 $\mu \mathrm{m}$ in rats and $20 \mu \mathrm{m}$ in macaques), was then applied to compensate for double counting in adjacent sections (Francois et al., 1999). 
5) In order to determine whether nigral $\mathrm{TH}+$ neurons are atrophic after PPN lesion, pictures of 60 Nissl-stained cell bodies of the SNc from 3 separate sections from 4 control macaques and of 3 Dtx-UII-lesioned animals were captured using a camera mounted to the microscope under a X 40 objective. The measure was performed on Nissl-stained cell bodies because thick $\mathrm{TH}+$ dendrites emerging from soma render the delineation of the cell bodies difficult. The cell body contours were traced and their surface was measured using Image $\mathbf{J}$ software. The data were then compiled and analyzed using Excel software.

6) TH-negative cell bodies in the $\mathrm{SNc}$ of sham- and Dtx-UII-lesioned rats were quantified on TH sections counterstained with cresyl violet, as this Nissl-stain contrasts well with the brown TH staining. The SNc was delineated in five regularly spaced sections whose levels were identical between animals, and we quantified large Nissl-stained cell bodies which were readily distinguishable from small glial cells. NADPH-negative cell bodies in the PPN of sham- and 6-OHDA-lesioned rats were also quantified on NADPH sections counterstained with neutral red, as this Nissl-stain contrasts well with the blue NADPH staining. Large neutral red-stained cell bodies were plotted in the PPN, delineated by the presence of $\mathrm{NADPH}+$ neurons in 5 sections whose levels were identical between animals.

\section{Data analysis}

All values are expressed as mean \pm SEM. All statistical analyses were performed using SigmaPlot (Systat Software, San Jose, CA). Side-to-side comparisons within the same group were made by Wilcoxon paired-sample. The non-parametric Kruskall-Wallis test was used to analyze variance among three groups followed by a Mann-Whitney $U$ test in the event of statistically significant differences. The non-parametric Mann-Whitney $U$ test was used for comparison of two groups (MPTP-treated and control macaques; 6-OHDA-lesioned-7w and 6-OHDA-lesioned-15w). Correlations between surviving $\mathrm{TH}+$ neurons in the $\mathrm{SNc}$ and 
$\mathrm{NADPH}+$ neurons in the PPN were analyzed using Pearson's correlation coefficient. The ttest was then used to establish if the correlation coefficient was significantly different from zero. The minimum level of statistical significance was set at $p<0.05$.

\section{Results}

\section{Comparison between control and sham-lesioned rats}

We first verified that saline injection into the right striatum or into the PPN did not induce neuronal loss. No difference was observed in the optical density of TH+ fibers in the striatum and the number of $\mathrm{TH}+$ neurons in the $\mathrm{SNc}$ ipsilateral to the sham lesion $(n=5$ hemispheres) compared to the contralateral side of the same animals, and compared to both hemispheres of non-operated, control rats ( $n=4$ hemispheres) (not shown). Likewise, the number of NADPH+ neurons was not affected by saline injections. Therefore, sham-lesioned and control animals were pooled together for subsequent analyses and were considered as the sham-lesioned group ( $n=7$ rats) for this study.

\section{Effect of cholinergic PPN lesion on nigral DA neuronal loss in rats}

Only rats with a more than $20 \%$ reduction of cholinergic neurons in the PPN were included in the analysis. The Dtx-UII-lesioned group was thus composed of 5 rats with unilateral PPN lesion and 3 rats with bilateral PPN lesion ( $n=11$ hemispheres) that survived 7 weeks. Data are expressed as total numbers of TH+ and NADPH+ neurons per hemisphere of rats.

Injections of Dtx-UII into the PPN of rats induced a mean loss of $33 \%$ of NADPH+ neurons, mainly centrally located, compared with the sham-lesioned group $(p<0.001$, Kruskall-Wallis) (Fig. 2A, Table 1). Examination of NeuN-stained PPN sections of rats with Dtx-UII lesion showed that the major effect of Dtx-UII administration was on cholinergic 
neurons, with little damage to non-cholinergic neurons, which was mainly confined to the needle tracks (Fig. 1B,D). This result confirms that Dtx-UII used at 5\%, thus higher than previously used in the initial report that described this protocol (3\%), remains selective for cholinergic PPN neurons (Clark et al., 2007). These cholinergic lesions resulted in a significant loss of $14 \%$ of $\mathrm{TH}+$ fibers in the striatum (Fig. 2B) and of $26 \%$ of $\mathrm{TH}+$ neurons in the SNc when compared to the sham-lesioned group ( $p<0.005$, Kruskall-Wallis) (Fig. 2C,D). These losses were found in various sectors of the $\mathrm{SNc}$ and striatum different from one lesioned animal to another and most likely depending on the precise localization of the PPN lesion. No TH+ cell loss was observed contralaterally to the unilateral lesion of the PPN.

To investigate the possibility that the reduction in cell numbers was simply due to a loss of TH expression, $5 \mathrm{TH}+$ sections of sham- and Dtx-UII-lesioned rats were counterstained with cresyl violet in order to visualize cell bodies in the SNc (Fig. 2E). A larger number of neuronal counterstained cell bodies that were TH-negative was found in the SNc of the DtxUII-lesioned side compared to the sham-lesioned side (+8\%; Dtx-UII: $1822 \pm 51$ cell bodies; sham: $1683 \pm 48$ cell bodies), but this difference was not significant ( $p=0.18$, Mann-Whitney $U$ test). This suggests that the reduction in TH cell number after the PPN lesion was not due to a loss of TH expression but to actual neuronal cell loss.

To determine if the PPN lesion induced inflammation at the nigral level, the number of Iba-1+ microglial cells was quantified in the SNc of Dtx-UII-lesioned rats 7 weeks after injection and compared to sham-lesioned rats. The number of Iba-1+ cells were similar to the sham group (Dtx-UII: $86.9 \pm 4.5$ cells per $\mathrm{mm}^{2}$; sham: $82.1 \pm 3.7$ cells per $\mathrm{mm}^{2} ; p=0.62$, Mann-Whitney $U$ test) and were homogeneously distributed. No activated microglial cells, characterized by round amoeboid-shaped cells, were identified. To investigate if a proinflammatory state may have occurred earlier in the process of degeneration, the number of Iba-1+ cells was quantified in the SNc of PPN-lesioned rats 3 weeks after injection, thus when 
the Dtx-UII toxin had induced maximal loss of cholinergic neurons (Clark et al., 2007). We first verified that Dtx-UII lesion in the PPN induced a significant loss of $39 \%$ of NADPH+ neurons compared with the sham-lesioned group (Dtx-UII 3 weeks: $1521 \pm 191$; sham: 2474 \pm 33 neurons; $p<0.05$, Mann-Whitney $U$ test). However, no statistical difference was observed in the number of nigral $\mathrm{TH}+$ neurons and of Iba- $1+$ cells between the Dtx-UIIlesioned group 3 weeks post-lesion and the sham-lesioned group (number of $\mathrm{TH}+$ neurons: Dtx-UII 3 weeks: 10,956 \pm 183 neurons; sham: $11,911 \pm 182$ neurons; $p=0.06$, MannWhitney $U$ test. Number of Iba1+ cells: Dtx-UII 3 weeks: $85.2 \pm 4.5$ cells per $\mathrm{mm}^{2}$; sham: $82.1 \pm 3.7$ cells per $\mathrm{mm}^{2} ; p=0.75$, Mann-Whitney $U$ test). Only a very few round amoeboidshaped Iba1+ cells were identified in the SNc of the Dtx-UII-lesioned group 3 weeks postlesion (Fig. 2F). This suggests that the PPN lesion did not induce a pro-inflammatory response at the nigral level.

\section{Effect of cholinergic PPN lesion on nigral DA neuronal loss in macaques}

A bilateral stereotaxic injection of Dtx-UII into the PPN of 5 macaques induced a loss of $39 \%$ of NADPH+ neurons compared to control animals $(n=5)(p<0.01$, Mann-Whitney $U$ test), as already reported (Karachi et al., 2010). There was no difference in the number of $\mathrm{TH}+$ fibers in the putamen and of $\mathrm{TH}+$ neurons in the SNc compared to control animals ( $p>0.05$, Mann-Whitney $U$ test) (Fig. 3A,A'; Table 1).

While no nigral $\mathrm{TH}+$ cell loss was detected in Dtx-UII-lesioned macaques, a $15 \%$ decrease in the surface area of Nissl-stained cell bodies was observed in all parts of the SNc (control, $479 \pm 50 \mu \mathrm{m}^{2}$; Dtx-UII, $407 \pm 45 \mu \mathrm{m}^{2}, p<0.001$, Mann-Whitney $U$ test) (Fig. $\left.3 \mathrm{~B}, \mathrm{~B}^{\prime}\right)$. Moreover, the greater the severity of the PPN lesion, the greater the reduction in cell body size. In contrast to what was observed in control animals, a few Fluoro-Jade-positive 
neurons were present in the SNc of macaques (Fig. 3C), suggesting that these neurons were degenerating.

\section{Effect of DA lesion on cholinergic PPN neuronal loss in rats}

The effects of DA lesions on nigral DA and PPN cholinergic neurons were first assessed separately before testing whether adding a DA lesion to a PPN cholinergic lesion would aggravate DA degeneration.

The efficacy of the 6-OHDA lesion in the right striatum of rats was first assessed by TH immunoreactivity in 6-OHDA-lesioned rats 7 weeks post-injection (6-OHDA-lesioned-7w rats). There were significant losses of $51 \%$ of $\mathrm{TH}+$ fibers in the striatum (Fig. $4 \mathrm{~A}$ ) and of $72 \%$ of $\mathrm{TH}+$ neurons in the SNc ipsilateral to the lesion compared with the sham-lesioned group ( $p<0.001$, Kruskall-Wallis) (Fig. 4B). A small but significant loss of $10 \%$ of TH+ neurons was also observed in the contralateral hemisphere ( $p<0.05$, Kruskall-Wallis, Fig. 3B). Unexpectedly, DA striatal lesions induced a significant loss of $29 \%$ of NADPH+ neurons in the PPN ipsilateral to the DA lesion ( $p<0.001$, Kruskall-Wallis; Fig. 4C).

To determine the effect of a longer survival time after 6-OHDA striatal lesion on NADPH neuronal loss, 5 rats were allowed to survive 15 weeks after 6-OHDA striatal lesion (6-OHDA-lesioned-15w rats). Compared with the sham-lesioned group, we observed a significant loss of $\mathrm{TH}+$ fibers in the striatum $(41 \%)$, and of $\mathrm{TH}+$ neurons in the $\mathrm{SNc}$ ipsilateral to the striatal 6-OHDA lesion (75\%) $(p<0.005$ and $p<0.001$, respectively, KruskallWallis) (Fig. 4A,B,D). A significant TH+ cell loss was also observed contralaterally (18\%) $(p<0.05)$. These DA lesions induced a significant difference in the number of NADPH+ neurons ipsilateral to the striatal lesion $(34 \%$ loss, $p<0.05)$ but also contralaterally $(11 \%$ loss, $p<0.005$ ) (Fig. 4C,D). The NADPH+ neuronal degeneration was greater after 15 weeks of 
survival time than after 7 weeks, but the difference was not significant. Moreover, the loss of $\mathrm{NADPH}+$ neurons was higher in the anterior PPN than in its posterior part (Fig. 4E).

To investigate the possibility that the reduction in cell numbers was solely due to a loss of NADPH diaphorase expression, NADPH+ cell bodies were quantified on 5 sections counterstained with neutral red. The number of neuronal cell bodies that were neutral red stained and NADPH-negative increased in the PPN of 6-OHDA-lesioned rats compared to sham-lesioned $(+39 \%, p<0.01)$ (Fig. 4F). This suggests that these NADPH-negative neurons correspond to cholinergic neurons that did not fully degenerate after 6-OHDA lesion but lost their ability to express NADPH diaphorase.

Examination of PPN sections stained with the microglial marker Iba-1 and ChAT revealed no inflammation in the PPN of Dtx-UII-lesioned groups compared to sham-lesioned animals, except along the needle tracks (Fig. 1A).

\section{Effect of DA lesion on cholinergic PPN neuronal loss in macaques}

The effect of a DA lesion on cholinergic neuronal survival was then assessed in MPTPtreated macaques. MPTP induced a massive bilateral loss of $81 \%$ of $\mathrm{TH}+$ neurons in the $\mathrm{SNc}$ and of $78 \%$ of $\mathrm{TH}+$ fibers in the striatum $(p<0.005$, Mann-Whitney $U$ test $)$, and a small but significant loss of $7.4 \%$ of NADPH+ neurons in the PPN of both hemispheres $(p<0.005$, Mann-Whitney $U$ test) (Table 1 and Fig. 5). No reduction in cell body size of NADPH+ neurons was observed in MPTP-treated macaques when compared with control animals.

\section{Effect of simultaneous lesion of both DA and cholinergic neurons on neuronal losses in rats}

PPN cholinergic lesions were performed successively in the two hemispheres one week and two weeks, respectively, after the unilateral 6-OHDA lesion, i.e. at a stage when DA neurons were degenerating. These doubly lesioned animals showed a reduction of $52 \%$ in 
$\mathrm{TH}+$ fibers in the striatum, and of $84 \%$ in $\mathrm{TH}+$ neurons in the $\mathrm{SNc}$ ipsilateral to the lesion compared with sham-lesioned rats $(p<0.001)$ and $23 \%$ in $\mathrm{TH}+$ nigral neurons contralaterally $(p<0.01)$ (Fig. 6A,B). TH degeneration was therefore slightly more extensive in the double lesioning paradigm than after a single lesion of the nigrostriatal pathway, but the difference was not significant ( $p>0.05$, test a posteriori). These doubly lesioned animals also showed a reduction in NADPH+ neurons of $53 \%$ ipsilaterally and of $27 \%$ contralaterally to the PPN cholinergic lesion ( $p<0.001$ and $p<0.05$, respectively, Kruskall-Wallis) (Fig. 6C,D), which was greater than the reduction observed after a single PPN cholinergic lesion $(p<0.05)$.

Correlation between the intensity of DA neuronal degeneration and the extent of cholinergic PPN neuronal loss in rats

A strong correlation between $\mathrm{TH}+$ and $\mathrm{NADPH}+$ neuronal losses was observed in all experimental conditions in rats. The correlation coefficient was 0.83 for rats with PPN lesion (Dtx-UII-lesioned rats), 0.88 for rats with DA striatal lesion (6-OHDA-lesioned rats), 0.90 for double-lesioned rats (Fig. 7) and 0.78 for all groups of rats. In each case the correlation coefficient was statistically significant $(p<0.001)$.

\section{Discussion}

The major findings of the present study are that 1) lesioning of PPN cholinergic neurons resulted in a significant loss of DA neurons in the $\mathrm{SNc}$ of rats and atrophy of nigral DA neurons in macaques, 2) a DA lesion induced a significant loss of PPN cholinergic neurons in both rats and monkeys, and 3) adding PPN cholinergic lesion in 6-OHDA-lesioned rats when DA nigral neurons were degenerating resulted in more drastic DA and cholinergic neuronal losses than when each neurotransmitter system was lesioned separately. 
Our findings demonstrate that partially removing excitatory cholinergic inputs to DA nigral neurons as observed in the course of PD results in DA neuronal dysfunction. Several hypotheses might be considered to explain the dysfunction and death of DA neurons after the cholinergic PPN lesion. It is first possible that the cholinergic lesion caused overactivity of glutamatergic PPN neurons that also project to DA neurons (Charara et al., 1996), resulting in an excitotoxic stimulation of DA neurons and ultimately their death. This could explain why PPN lesioning before MPTP intoxication attenuates DA cell loss and parkinsonian deficits compared with MPTP-treated macaques (Takada et al., 2000). In that sense, it would be of interest to determine the activity of glutamatergic PPN neurons after cholinergic PPN lesion by performing electrophysiological recordings on these neurons, or by quantifying cellular levels of cytochrome oxidase subunit I mRNAs. Alternatively, the decrease of cholinergic inputs to DA neurons may result in a reduced activity and excitability of these neurons, leading progressively to their suffering and demise (Michel et al., 2013). This is in line with the atrophy of DA nigral neurons that we observed in PPN-lesioned macaques, since cell shrinkage and the presence of Fluoro-Jade-positive neurons are characteristic hallmarks of apoptosis (Maeno et al., 2000). This is coherent with the fact that the rate-limiting enzyme in dopamine synthesis, tyrosine hydroxylase, whose expression is activity- and calciumdependent (Brosenitsch and Katz, 2001), is absent from a large fraction of neuromelanized DA neurons in PD brains (Hirsch et al., 1988). Some reports also suggest that $\alpha 7$ nicotinic receptor activation provides protection to DA neurons in midbrain cultures (Toulorge et al., 2011) and in rodent models of PD (Suzuki et al., 2013; Bordia et al., 2015), whereas a selective $\alpha 7 \mathrm{nAChR}$ antagonist suppressed these effects (Suzuki et al., 2013). Moreover, stimulating the PPN increases the firing rate and electrical activity of DA neurons (Clarke et al., 1987; Forster and Blaha, 2003). 
Lesioning of PPN cholinergic neurons with Dtx-UII in macaques resulted in lesions that were of similar extent as in rats (33\% in rats and $39 \%$ in macaques). Surprisingly, the PPN cholinergic lesion resulted in a loss of DA neurons in the SNc of rats but only in the atrophy of these neurons in macaques. This discrepancy is difficult to explain, but is consistent with the different results of electrophysiological studies in rodent, non-human primate and human patients with Parkinson's disease, which may be explained on the basis of variable afferent and efferent connectivities of these nuclei to the basal ganglia (Alam et al., 2010, review). Thus, it has been suggested that cholinergic neurons account for $25 \%$ of all PPN neurons projecting to the SNc in monkeys (Lavoie and Parent, 1994) and 40\% in rodents (Woolf and Butcher, 1986; Gould et al., 1989). It is thus possible that pure cholinergic PPN lesions were not sufficient to induce DA degeneration in primates, and that removing both glutamatergic and cholinergic inputs to DA neurons may be necessary to reduce activity of these neurons. This may explain why only extensive PPN lesions involving both cholinergic and noncholinergic neurons induced a parkinsonian syndrome in macaques (Aziz et al., 1998; Matsumura and Kojima, 2001). PPN lesions in rats also resulted in a decreased activity of SNc neurons (Breit et al., 2005), in the development of oxidative stress events in the SNc (Jimenez-Martin et al., 2015) and in DA neuronal death when the lesion is extensive (McGeer and McGeer, 1984). These observations suggest that basal excitatory glutamatergic inputs may themselves contribute to the survival of DA neurons. This is, however, rather unlikely even if the activation of glutamate N-methyl-D-aspartate receptors was reported to contribute to the maintenance of DA neurons in rat midbrain slice cultures (Katsuki et al, 2003).

Unilateral 6-OHDA striatal lesion in rats resulted in a reduced number of $\mathrm{TH}+$ neurons not only ipsilaterally but also contralaterally to the lesion, the reduction being greater 15 weeks than 7 weeks post-lesion. Unilateral 6-OHDA lesion has already been reported to 
induce contralateral loss of DA neurons (Yuan et al., 2005) and a decrease of striatal DA levels (Hahn et al., 1981), as well as bilateral structural alterations in the SN and cortex detected using magnetic resonance imaging (Soria et al., 2011). This suggests that the side contralateral to the DA lesion should no longer be considered as a control side. Moreover, the loss of DA fibers in the striatum 7 weeks post-lesion tended to diminish after 15 weeks, which is consistent with previous studies reporting a partial recovery of DA striatal fibers between 5 and 8 weeks (Yuan et al., 2005) and a more substantial recovery 16 weeks (Zeng et al., 2012) after striatal 6-OHDA lesion. This may also explain why no decrease in optical density for TH was observed contralaterally to the lesion whereas the number of $\mathrm{TH}+$ neurons and NADPH+ neurons was significantly decreased. Moreover, these results indicate that the remaining DA neurons could sprout and re-innervate the striatum.

Our findings show that DA degeneration produced a statistically significant reduction in PPN cholinergic neurons in rats, the damage evolving over time ipsilaterally and contralaterally to the lesion. Our results are consistent with the observation that 6-OHDA lesion in rats alters PPN spike timing (Aravamuthan et al., 2008). Furthermore, a DA lesion induced by intranigral injection of the proteasome inhibitor lactacystin in rats also resulted in a $61 \%$ loss of PPN cholinergic neurons ipsilateral to the lesion, accompanied by somatic hypotrophy in the remaining cholinergic neurons (Pienaar et al., 2015). Note that in the present study the extent of DA neuronal loss in rats was also strongly correlated with that of cholinergic PPN neurons, as reported in PD patients (Zweig et al., 1989; Karachi et al., 2010). In macaques, however, extensive DA degeneration was associated with a limited PPN cholinergic lesion. Differences observed here in the extent of cholinergic neuronal loss between rats and macaques may be due either to differences in the mechanisms involved in MPTP and 6-OHDA toxicity, or to the more progressive DA neuronal loss induced by a subchronic MPTP regimen (1 to 2 months) than that induced by intrastriatal 6-OHDA injection (2 
to 3 weeks post-infusion) (Sauer and Oertel, 1994; Blandini et al., 2007), allowing compensatory mechanisms to develop in the PPN of monkeys. The existence of plasticity, such as trophic compensation at striatal level after MPTP treatment, has been evidenced in young macaques, but not in old animals (Collier et al., 2005). This raises the possibility that various insults may progressively develop with time, which is consistent with the observation that PPN cholinergic neurons are lost only in aged MPTP-treated monkeys (Karachi et al., 2010). We thus hypothesize that a longer time-scale was required for the development of a cholinergic lesion in monkeys, and that similar mechanisms may be involved in the pathogenesis of PD.

An absence of cholinergic PPN lesion was reported in rat and macaque models of PD similar to those used in the present study (Herrero et al., 1993; Heise et al., 2005; Pienaar and van de Berg, 2013). Several factors could account for these discrepancies. 1) The low number of macaques used in previous studies is perhaps insufficient to detect a cholinergic neuronal loss, which was modest (7\%) in our material and statistically significant only when a sixth animal was added. 2) Apomorphine, known for its neurotrophic and neuroprotective effect (Yuan et al., 2004) and given to test behaviorally the 6-OHDA-lesioned rats in other studies but not in our case, may also account for the attenuation of DA degeneration. 3) Quantifying cholinergic and DA neurons using stereology as we performed is more accurate than the other methods used in other studies. 4) Lastly, the side contralateral to the 6-OHDA lesion, although affected by the lesion, was used as a control side by the different research teams. A recent study also reported that loss of DA neurons does not affect cholinergic neuron numbers in the PPN of rats (Gut and Winn, 2015). The rat strain (Lister Hooded) and the short survival time after 6-OHDA lesion (3 weeks) used in this study may explain discrepancies in cholinergic PPN neuronal vulnerability. The fact that no cholinergic cell death was detected in this study does not exclude the possibility that cholinergic neurons were dysfunctional. 
Several hypotheses might be considered to explain why DA degeneration resulted in cholinergic PPN lesion. 1) PPN neurons are hyperactive in unilaterally 6-OHDA-lesioned rats but not in rats with combined subthalamic and DA lesions, which suggests that the PPN is under major control of the subthalamic nucleus (Breit et al., 2001). The subthalamic glutamatergic neurons being hyperactive in the parkinsonian state, this hyperactivity may cause the overexcitation of PPN cholinergic neurons through the sustained engagement of NMDA glutamate receptors, leading ultimately to excitotoxic death of cholinergic neurons. 2) Alternatively, the hyperactivity of GABAergic outputs from the pallidum and SN pars reticulata observed after DA lesion may result in excessive inhibition of PPN neurons, as reported in MPTP-treated macaques (Gomez-Gallego et al., 2007). The reduced activity of PPN neurons may result in progressive cell death, as demonstrated for DA neurons (Michel et al., 2013). However, this remains speculative and needs to be determined. 3) It has been proposed that the loss of DA neurons in PD may occur via a "dying back" process that begins in striatal nerve terminals and terminates at the somatic level (Morfini et al., 2009; Chu et al., 2012). It is possible that PPN neuronal loss might have followed a similar mode of degeneration in 6-OHDA-lesioned rats, as previously suggested (Pienaar and van de Berg, 2013), possibly due to the deafferentation process that affects cholinergic nerve terminals.

\section{Conclusions}

Overall, our data 1) confirm the important correlation that exists between the extent of loss of DA neurons in the SN and that of cholinergic neurons in the PPN (Zweig et al., 1989), and 2) demonstrate that reciprocal interactions between cholinergic PPN neurons and SN DA neurons make these populations of neurons mutually dependent on each other for their survival. Moreover, adding PPN lesion in the course of the degenerative process affecting DA fibers in rats induced more drastic losses of both cholinergic PPN and DA nigral neurons than 
when each neurotransmitter system was lesioned separately. This further suggests that a vicious circle type of degeneration involving the SN DA and PPN cholinergic systems may develop as PD-related events progress. Our results thus point towards a key role of PPN cholinergic neurons in the pathophysiology of PD. Whether cholinergic PPN degeneration is the primary cause or the consequence of DA lesions remains, however, to be determined. 


\section{References}

Alam, M., Schwabe, K., Krauss, J.K., 2011. The pedunculopontine nucleus area: critical evaluation of interspecies differences relevant for its use as a target for deep brain stimulation. Brain 134, 11-23.

Aravamuthan, B.R., Bergstrom, D.A., French, R.A., Taylor, J.J., Parr-Brownlie, L.C., Walters, J.R., 2008. Altered neuronal activity relationships between the pedunculopontine nucleus and motor cortex in a rodent model of Parkinson's disease. Exp. Neurol. 213, 268280.

Aravamuthan, B.R., Muthusamy, K.A., Stein, J.F., Aziz, T.Z., Johansen-Berg, H., 2007. Topography of cortical and subcortical connections of the human pedunculopontine and subthalamic nuclei. Neuroimage 37, 694-705.

Aziz, T.Z., Davies, L., Stein, J., France, S., 1998. The role of descending basal ganglia connections to the brain stem in parkinsonian akinesia. Br. J. Neurosurg. 12, 245-249.

Blaha, C.D., Allen, L.F., Das, S., Inglis, W.L., Latimer, M.P., Vincent, S.R., Winn, P., 1996. Modulation of dopamine efflux in the nucleus accumbens after cholinergic stimulation of the ventral tegmental area in intact, pedunculopontine tegmental nucleus-lesioned, and laterodorsal tegmental nucleus-lesioned rats. J. Neurosci. 16, 714-722.

Blandini, F., Levandis, G., Bazzini, E., Nappi, G., Armentero, M.T., 2007. Time-course of nigrostriatal damage, basal ganglia metabolic changes and behavioural alterations following intrastriatal injection of 6-hydroxydopamine in the rat: new clues from an old model. Eur. J. Neurosci. 25, 397-405.

Bordia, T., McGregor, M., Papke, R.L., Decker, M.W., Michael McIntosh, J., Quik, M., 2015. The alpha7 nicotinic receptor agonist ABT-107 protects against nigrostriatal damage in rats with unilateral 6-hydroxydopamine lesions. Exp. Neurol. 263, 277-284. 
Breit, S., Bouali-Benazzouz, R., Benabid, A.L., Benazzouz, A. 2001. Unilateral lesion of the nigrostriatal pathway induces an increase of neuronal activity of the pedunculopontine nucleus, which is reversed by the lesion of the subthalamic nucleus in the rat. Eur. J. Neurosci. 14, 1833-1842.

Breit, S., Lessmann, L., Benazzouz, A., Schulz, J.B., 2005. Unilateral lesion of the pedunculopontine nucleus induces hyperactivity in the subthalamic nucleus and substantia nigra in the rat. Eur. J. Neurosci. 22, 2283-2294.

Brosenitsch, T.A., Katz, D.M., 2001. Physiological patterns of electrical stimulation can induce neuronal gene expression by activating N-type calcium channels. J. Neurosci. 21, 2571-2579.

Charara, A., Smith, Y., Parent, A., 1996. Glutamatergic inputs from the pedunculopontine nucleus to midbrain dopaminergic neurons in primates: Phaseolus vulgaris-leucoagglutinin anterograde labeling combined with postembedding glutamate and GABA immunohistochemistry. J. Comp. Neurol. 364, 254-266.

Chu, Y., Morfini, G.A., Langhamer, L.B., He, Y., Brady, S.T., Kordower, J.H., 2012. Alterations in axonal transport motor proteins in sporadic and experimental Parkinson's disease. Brain 135, 2058-2073.

Clark, S.D., Alderson, H.L., Winn, P., Latimer, M.P., Nothacker, H.P., Civelli, O., 2007. Fusion of diphtheria toxin and urotensin II produces a neurotoxin selective for cholinergic neurons in the rat mesopontine tegmentum. J. Neurochem. 102, 112-120.

Clarke, P.B., Hommer, D.W., Pert, A., Skirboll, L.R., 1987. Innervation of substantia nigra neurons by cholinergic afferents from pedunculopontine nucleus in the rat: neuroanatomical and electrophysiological evidence. Neuroscience. 23, 1011-1019. 
Collier, T.J., Dung Ling, Z., Carvey, P.M., Fletcher-Turner, A., Yurek, D.M., Sladek, J.R. Jr, Kordower, J.H., 2005. Striatal trophic factor activity in aging monkeys with unilateral MPTP-induced parkinsonism. Exp. Neurol. 191, S60-67.

Costa, G., Abin-Carriquiry, J.A., Dajas, F., 2001. Nicotine prevents striatal dopamine loss produced by 6-hydroxydopamine lesion in the substantia nigra. Brain Res. 888, 336-342.

Forster, G.L., Blaha, C.D., 2003. Pedunculopontine tegmental stimulation evokes striatal dopamine efflux by activation of acetylcholine and glutamate receptors in the midbrain and pons of the rat. Eur. J. Neurosci. 17, 751-762.

Francois, C., Yelnik, J., Tandé, D., Agid, Y., Hirsch, E.C., 1999. Dopaminergic cell group A8 in the monkey: anatomical organization and projections to the striatum. J. Comp. Neurol. $414,334-347$

Geula, C., Schatz, C.R., Mesulam, M.M., 1993. Differential localization of NADPHdiaphorase and calbindin-D28k within the cholinergic neurons of the basal forebrain, striatum and brainstem in the rat, monkey, baboon and human. Neuroscience. 54, 461-476.

Gomez-Gallego, M., Fernandez-Villalba, E., Fernandez-Barreiro, A., Herrero, M.T., 2007. Changes in the neuronal activity in the pedunculopontine nucleus in chronic MPTP-treated primates: an in situ hybridization study of cytochrome oxidase subunit I, choline acetyl transferase and substance P mRNA expression. J. Neural. Transm. 114, 319-326.

Gonzalez-Hernandez, T., Abdala, P., Rodriguez, M., 1997. NOS expression in nigral cells after excitotoxic and non-excitotoxic lesion of the pedunculopontine tegmental nucleus. Eur. J. Neurosci. 9, 2658-2667.

Gould, E., Woolf, N.J., Butcher, L.L., 1989. Cholinergic projections to the substantia nigra from the pedunculopontine and laterodorsal tegmental nuclei. Neuroscience. 28, 611-623. 
Grabli, D., Karachi, C., Folgoas, E., Monfort, M., Tande, D., Clark, S., Civelli, O., Hirsch, E.C., Francois, C., 2013. Gait disorders in parkinsonian monkeys with pedunculopontine nucleus lesions: a tale of two systems. J. Neurosci. 33, 11986-11993.

Gut, N.K., Winn, P., 2015. Deep brain stimulation of different pedunculopontine targets in a novel rodent model of parkinsonism. J. Neurosci. 35, 4792-4803.

Hahn, Z., Karadi, Z., Lenard, L., 1981. Striatal dopamine levels after unilateral lesions of the substantia nigra: evidence for a contralateral decrease. Acta Physiol. Acad. Sci. Hung. 57, 249-253.

Heise, C.E., Teo, Z.C., Wallace, B.A., Ashkan, K., Benabid, A.L., Mitrofanis, J., 2005. Cell survival patterns in the pedunculopontine tegmental nucleus of methyl-4-phenyl-1,2,3,6tetrahydropyridine-treated monkeys and 6OHDA-lesioned rats: evidence for differences to idiopathic Parkinson disease patients? Anat. Embryol. (Berl). 210, 287-302.

Hernan, M.A., Takkouche, B., Caamano-Isorna, F., Gestal-Otero, J.J., 2002. A meta-analysis of coffee drinking, cigarette smoking, and the risk of Parkinson's disease. Ann. Neurol. 52, 276-284.

Herrero, M.T., Hirsch, E.C., Javoy-Agid, F., Obeso, J.A., Agid, Y., 1993. Differential vulnerability to 1-methyl-4-phenyl-1,2,3,6- tetrahydropyridine of dopaminergic and cholinergic neurons in the monkey mesopontine tegmentum. Brain Res. 624, 281-285.

Hirsch, E., Graybiel, A.M., Agid, Y.A., 1988. Melanized dopaminergic neurons are differentially susceptible to degeneration in Parkinson's disease. Nature. 334, 345-348.

Hirsch, E.C., Graybiel, A.M., Duyckaerts, C., Javoy-Agid, F., 1987. Neuronal loss in the pedunculopontine tegmental nucleus in Parkinson disease and in progressive supranuclear palsy. Proc. Natl. Acad. Sci. U.S.A. 84, 5976-5980.

Jellinger, K., 1988. The pedunculopontine nucleus in Parkinson's disease, progressive supranuclear palsy and Alzheimer's disease. J. Neurol. Neurosurg. Psychiatry. 51, 540-543. 
Jimenez-Martin, J., Blanco-Lezcano, L., Gonzalez-Fraguela, M.E., Diaz-Hung, M.L., Serrano-Sanchez, T., Almenares, J.L., Francis-Turner, L., 2015. Effect of neurotoxic lesion of pedunculopontine nucleus in nigral and striatal redox balance and motor performance in rats. Neuroscience. $289,300-314$.

Karachi, C., Grabli, D., Bernard, F.A., Tandé, D., Wattiez, N., Belaid, H., Bardinet, E., Prigent, A., Nothacker, H.P., Hunot, S., Hartmann, A., Lehéricy, S., Hirsch, E.C., Francois, C., 2010. Cholinergic mesencephalic neurons are involved in gait and postural disorders in Parkinson disease. J. Clin. Invest. 120, 2745-2754.

Kojima, J., Yamaji, Y., Matsumura, M., Nambu, A., Inase, M., Tokuno, H., Takada, M., Imai, H., 1997. Excitotoxic lesions of the pedunculopontine tegmental nucleus produce contralateral hemiparkinsonism in the monkey. Neurosci. Lett. 226, 111-114.

Lavoie, B., Parent, A., 1994. Pedunculopontine nucleus in the squirrel monkey: cholinergic and glutamatergic projections to the substantia nigra. J. Comp. Neurol. 344, 232-241.

Maeno, E., Ishizaki, Y., Kanaseki, T., Hazama, A., Okada, Y., 2000. Normotonic cell shrinkage because of disordered volume regulation is an early prerequisite to apoptosis. Proc. Natl. Acad. Sci. U. S. A. 97, 9487-9492.

Matsumura, M., Kojima, J., 2001. The role of the pedunculopontine tegmental nucleus in experimental parkinsonism in primates. Stereotact. Funct. Neurosurg. 77, 108-115.

McGeer, E.G., McGeer, P.L., 1984. Substantia nigra cell death from kainic acid or folic acid injections into the pontine tegmentum. Brain Res. 298, 339-342.

Mesulam, M.M., Mufson, E.J., Wainer, B.H., Levey, A.I., 1983. Central cholinergic pathways in the rat: an overview based on an alternative nomenclature (Ch1-Ch6). Neuroscience 10, 1185-1201. 
Michel, P.P., Toulorge, D., Guerreiro, S., Hirsch, E.C., 2013. Specific needs of dopamine neurons for stimulation in order to survive: implication for Parkinson disease. FASEB J. 27, 3414-3423.

Morfini, G.A., Burns, M., Binder, L.I., Kanaan, N.M., LaPointe, N., Bosco, D.A., Brown, R.H., Jr., Brown, H., Tiwari, A., Hayward, L., Edgar, J., Nave, K.A., Garberrn, J., Atagi, Y., Song, Y., Pigino, G., Brady, S.T. 2009. Axonal transport defects in neurodegenerative diseases. J. Neurosci. 29, 12776-12786.

MunroDavies, L.E., Winter, J., Aziz, T.Z., Stein, J.F., 1999. The role of the pedunculopontine region in basal-ganglia mechanisms of akinesia. Exp.Brain Res. 129, 511-517.

Nastuk, M.A., Graybiel, A.M., 1991. Pharmacologically defined M1 and M2 muscarinic cholinergic binding sites in the cat's substantia nigra: development and maturity. Dev Brain Res. 61, 1-10.

Parain, K., Hapdey, C., Rousselet, E., Marchand, V., Dumery, B., Hirsch, E.C., 2003. Cigarette smoke and nicotine protect dopaminergic neurons against the 1-methyl-4-phenyl1,2,3,6-tetrahydropyridine Parkinsonian toxin. Brain Res. 984, 224-232.

Paxinos, G., Watson, C., 1998. The rat brain in stereotaxic coordinates, 4th ed. New York: Academic Press.

Pienaar, I.S., van de Berg, W., 2013. A non-cholinergic neuronal loss in the pedunculopontine nucleus of toxin-evoked parkinsonian rats. Exp. Neurol. 248, 213-223.

Pienaar, I.S., Harrison, I.F., Elson, J.L., Bury, A., Woll, P., Simon, A.K., Dexter, D.T., 2015. An animal model mimicking pedunculopontine nucleus cholinergic degeneration in Parkinson's disease. Brain Struct. Funct. 220, 479-500.

Quik, M., Perez, X.A., Bordia, T., 2012. Nicotine as a potential neuroprotective agent for Parkinson's disease. Mov. Disord. 27, 947-957. 
Salthun-Lassalle, B., Hirsch, E.C., Wolfart, J., Ruberg, M., Michel, P.P., 2004. Rescue of mesencephalic dopaminergic neurons in culture by low-level stimulation of voltage-gated sodium channels. J. Neurosci. 24, 5922-5930.

Sauer, H., Oertel, W.H., 1994. Progressive degeneration of nigrostriatal dopamine neurons following intrastriatal terminal lesions with 6-hydroxydopamine: a combined retrograde tracing and immunocytochemical study in the rat. Neuroscience. 59, 401-415.

Schmued, L.C., Hopkins, K.J., 2000. Fluoro-Jade B: a high affinity fluorescent marker for the localization of neuronal degeneration. Brain Res. 874, 123-130.

Sorenson, E.M., Shiroyama, T., Kitai, S.T., 1998. Postsynaptic nicotinic receptors on dopaminergic neurons in the substantia nigra pars compacta of the rat. Neuroscience. 87, 659-673.

Soria, G., Aguilar, E., Tudela, R., Mullol, J., Planas, A.M., Marin, C., 2011. In vivo magnetic resonance imaging characterization of bilateral structural changes in experimental Parkinson's disease: a T2 relaxometry study combined with longitudinal diffusion tensor imaging and manganese-enhanced magnetic resonance imaging in the 6-hydroxydopamine rat model. Eur. J. Neurosci. 33, 1551-1560.

Suzuki, S., Kawamata, J., Matsushita, T., Matsumura, A., Hisahara, S., Takata, K., Kitamura, Y., Kem, W., Shimohama, S., 2013. 3-[(2,4-Dimethoxy)benzylidene]-anabaseine dihydrochloride protects against 6-hydroxydopamine-induced parkinsonian neurodegeneration through alpha7 nicotinic acetylcholine receptor stimulation in rats. $\mathrm{J}$. Neurosci. Res. 91, 462-471.

Takada, M., Matsumura, M., Kojima, J., Yamaji, Y., Inase, M., Tokuno, H., Nambu, A., Imai, H., 2000. Protection against dopaminergic nigrostriatal cell death by excitatory input ablation. Eur. J. Neurosci. 12, 1771-1780. 
Thacker, E.L., O'Reilly, E.J., Weisskopf, M.G., Chen, H., Schwarzschild, M.A., McCullough, M.L., Calle, E.E., Thun, M.J., Ascherio, A., 2007. Temporal relationship between cigarette smoking and risk of Parkinson disease. Neurology. 68, 764-768.

Toulorge, D., Guerreiro, S., Hild, A., Maskos, U., Hirsch, E.C., Michel, P.P., 2011. Neuroprotection of midbrain dopamine neurons by nicotine is gated by cytoplasmic $\mathrm{Ca} 2+$. FASEB J. 25, 2563-2573.

West, M.J., Slomianka, L., Gundersen, H.J., 1991. Unbiased stereological estimation of the total number of neurons in the subdivisions of the rat hippocampus using the optical fractionator. Anat. Rec. 231, 482-497.

Woolf, N.J., Butcher, L.L., 1986. Cholinergic systems in the rat brain: III. Projections from the pontomesencephalic tegmentum to the thalamus, tectum, basal ganglia, and basal forebrain. Brain Res. Bull. 16, 603-637.

Yuan, H., Sarre, S., Ebinger, G., Michotte, Y., 2004. Neuroprotective and neurotrophic effect of apomorphine in the striatal 6-OHDA-lesion rat model of Parkinson's disease. Brain Res. 1026, 95-107.

Yuan, H., Sarre, S., Ebinger, G., Michotte, Y., 2005. Histological, behavioural and neurochemical evaluation of medial forebrain bundle and striatal 6-OHDA lesions as rat models of Parkinson's disease. J. Neurosci. Methods. 144, 35-45.

Zeng, X., Shen, H., Zhao, J., Cai, Q., Wang, C., Xu, Q., 2012. Long-term changes in morphology, D2R expression and targets of regenerated dopaminergic terminals in the striatum after a partial lesion in the substantia nigra in the rat. Brain Res. 1450, 166-173.

Zweig, R.M., Jankel, W.R., Hedreen, J.C., Mayeux, R., Price, D.L., 1989. The pedunculopontine nucleus in Parkinson's disease. Ann. Neurol. 26, 41-46. 


\section{Acknowledgments}

The research leading to these results has received funding from the program "Investissements d'avenir" ANR-10-IAIHU-06. The authors would like to thank Nick Barton for language editing.

Conflict of Interest: The authors declare no competing financial interests. 


\section{Figure legends}

Figure 1. Lesion analysis (A) Photomicrographs of triple-fluorescence staining for ChAT (red), Iba-1 (green) cells, and DAPI (blue) in a sham-lesioned and a PPN-lesioned rat, showing slight inflammation along the needle tracks. The white arrow indicates the location of needle tracks. (B) Photomicrographs of a sham-lesioned (left) and a PPN-lesioned rat (right) with NADPH and neuronal nuclei (NeuN) staining on adjacent sections showing the major effect of Dtx-UII administration on cholinergic neurons (NADPH), and its little effect on non-cholinergic neurons $(\mathrm{NeuN})$, mainly along needle tracks. Arrows indicate the most lateral part of the PPN.

Figure 2. Effect of Dtx-UII injections into the PPN of rats. Dtx-UII injections into the PPN induced loss of NADPH+ neurons (A), of TH+ fibers in the striatum (B) and of TH+ neurons in the SNc (C) seen on transverse sections. (D) Graphs showing the total number of NADPH+ neurons in the PPN, of $\mathrm{TH}+$ fibers in the striatum using optical density (OD) and of $\mathrm{TH}+$ neurons in the SNc of Dtx-UII-lesioned animals (Dtx-UII, $n=11$ hemispheres) compared with the sham-lesioned group (sham, $n=14$ hemispheres). (E) Photomicrographs of TH+ sections counterstained with cresyl violet showing large cresyl violet-stained cell bodies (blue arrows) in a sham-lesioned rat and in a Dtx-UII-lesioned rat. (F) Photomicrographs of Iba1+ cells in a sham-lesioned rat and in a Dtx-UII-lesioned rat. ***p<0.005; ****p<0.001, MannWhitney $U$ test. $\mathrm{Nb}$, number. The superior cerebellar peduncle (scp) has been delineated in A.

Figure 3. Effect of Dtx-UII injections into the PPN of macaques. (A) Photomicrograph of NADPH-labeled transverse sections showing Dtx-UII (Dtx-UII) injection site into the PPN compared with a control. (A') Graphic representation of the distribution of NADPH+ neurons 
in the PPN. (B) Photomicrograph of Nissl-stained transverse sections of the SNc illustrating the shrinkage of cell bodies after PPN lesion compared with a control. (B') Quantification of the surface area of Nissl-stained cell bodies, showing a decrease in macaques with Dtx-UIIlesioned PPN $(n=5)$ compared with controls $(n=5)$. (C) Photomicrograph of Fluoro-Jadestained cells in the SNc of two macaques with Dtx-UII-lesioned PPN. *** $p<0.005$, MannWhitney $U$ test. The superior cerebellar peduncle (scp) has been delineated in A.

Figure 4. Effect of 6-OHDA striatal injections in rats that were allowed to survive 7 weeks (7w) or 15 weeks (15w) post-lesion. Photomicrographs of transverse sections illustrating that 6-OHDA injections induced loss of TH+ fibers in the striatum (A), of TH+ neurons in the SNc (B), and of NADPH+ neurons in the PPN (C). The superior cerebellar peduncle (scp) has been delineated in C. (D) Graphs showing the loss of TH+ fibers in the striatum assessed by measuring optical density (OD), of TH+ neurons in the SNc and of NADPH neurons in the PPN of 6-OHDA-lesioned rats that survived 7 weeks $(6-O H D A-7 \mathrm{w}, n$ $=8$ ipsilateral and 8 contralateral hemispheres), 6-OHDA-lesioned rats that survived 15 weeks (6-OHDA-15w, $n=5$ ipsilateral and 5 contralateral hemispheres) compared with the sham-lesioned group (sham, $n=14$ hemispheres). The neuronal degeneration ipsilateral (ipsi) to the lesion was statistically significant, and was greater after 15 weeks of survival time than after 7 weeks, but the difference was not statistically significant. (E) Graph showing that the loss of NADPH+ neurons expressed as a percentage in each of the 5 PPN sections was higher anteriorly (ant) than posteriorly (post). (F) Photomicrographs of NADPH+ sections counterstained with neutral red showing large, neutral red-stained cell bodies (red arrows) in a 6-OHDA-lesioned rat that survived 7 weeks, and not in a sham-lesioned rat. ${ }^{*} p<0.05$, $* * * p<0.005 ; * * * * p<0.001$, Kruskall-Wallis test. 
Figure 5. Effect of MPTP intoxication in macaques. (A) Photomicrograph of a TH+ transverse section showing a decrease in $\mathrm{TH}+$ neurons in the $\mathrm{SNc}$ of an MPTP-treated macaque compared with a control. (B) Left, graphic representation of the distribution of TH+ neurons in the SNc showing the severe DA neuronal loss. Right, quantification of the total number of NADPH+ neurons, showing a slight, but statistically significant decrease in MPTP-treated macaques $(n=6)$ compared with controls $(n=5)$. *** $p<0.005$, Mann-Whitney $U$ test.

Figure 6. Effect of a double cholinergic and DA lesion in rats. Photomicrographs of transverse sections illustrating that 6-OHDA striatal injections and Dtx-UII PPN injections resulted in the loss of $\mathrm{TH}+$ fibers in the striatum $(\mathbf{A})$, of $\mathrm{TH}+$ neurons in the $\mathrm{SNc}(\mathbf{B})$, and of NADPH+ neurons in the PPN (C). (D) Graphs showing loss of TH+ fibers in the striatum assessed by measuring optical density (OD) of TH+ neurons in the SNc and of NADPH+ neurons in the PPN of double-lesioned animals $(n=4$ ipsilateral and 4 contralateral hemispheres) compared with the sham-lesioned group ( $\mathrm{n}=14$ hemispheres). ${ }^{*} p<0.05$, $* * * * p<0.001$, Kruskall-Wallis test. The superior cerebellar peduncle (scp) has been delineated in $\mathbf{C}$.

Figure 7. Correlation between the number of TH+ neurons and the number of NADPH+ neurons. A strong correlation between the number of $\mathrm{TH}+$ neurons and the number of $\mathrm{NADPH}+$ neurons was observed in all experimental conditions in rats. Contra, contralateral; ipsi, ipsilateral. 


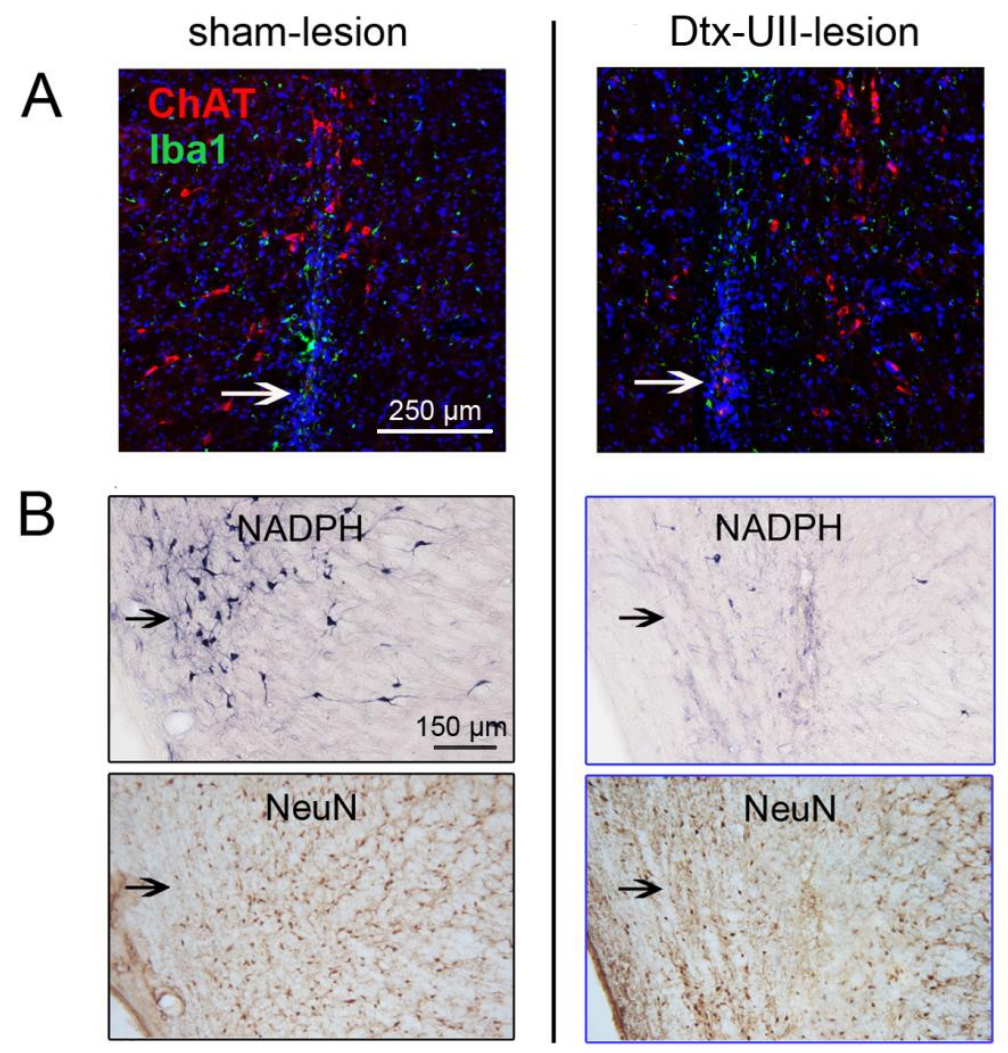

Fig. 1 


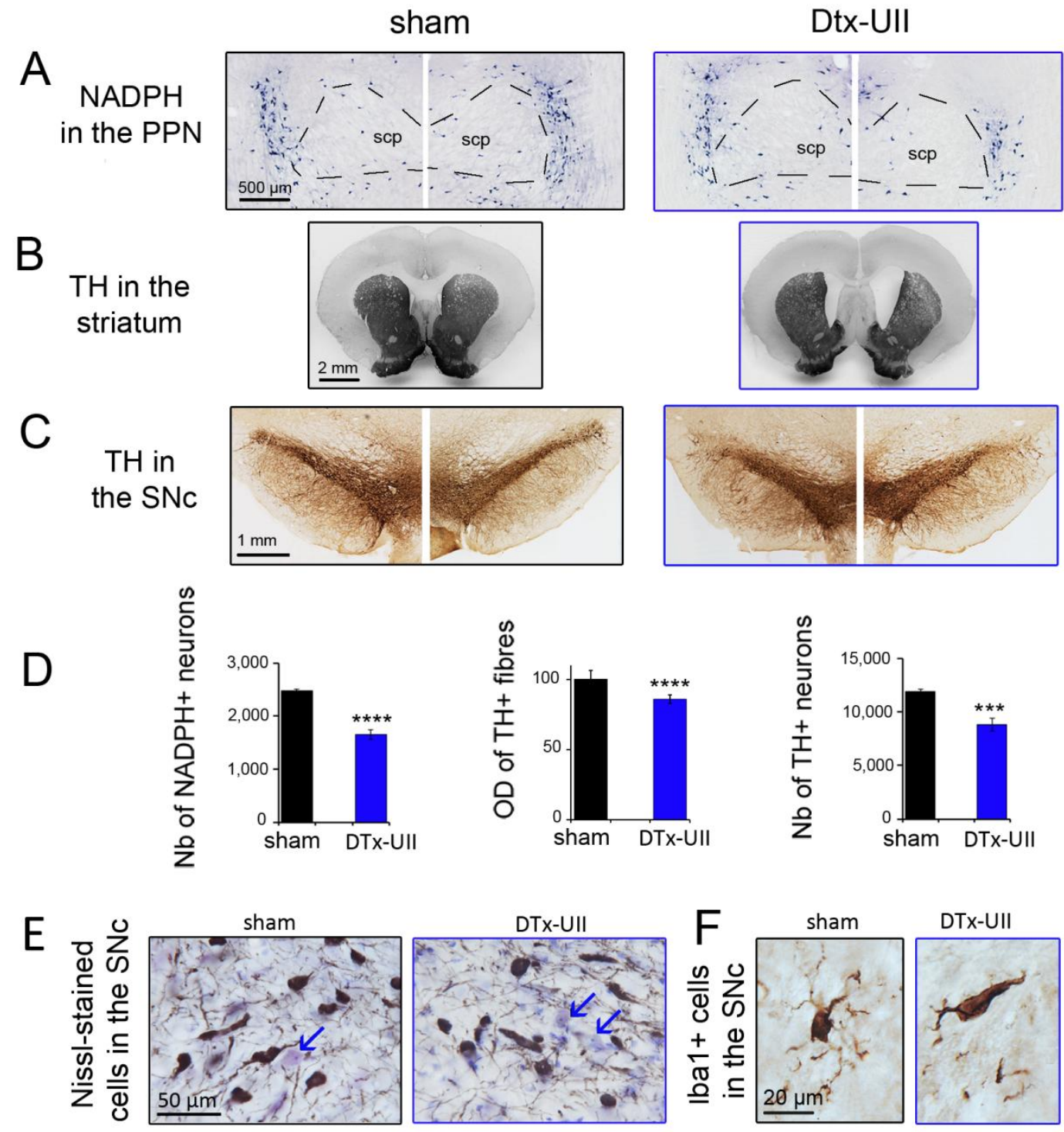

Fig. 2 

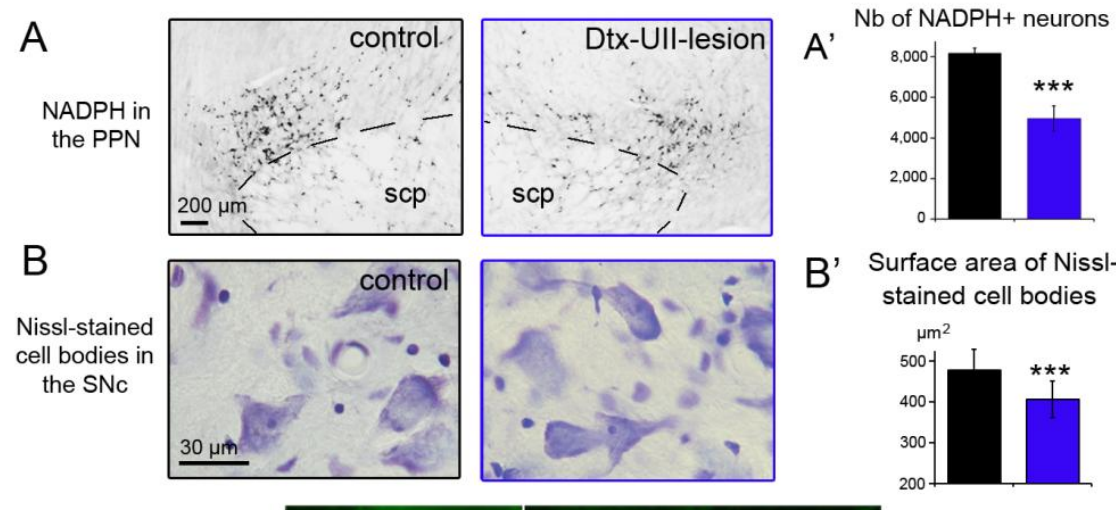

B' Surface area of Nisslstained cell bodies

C

Fluoro-Jade B

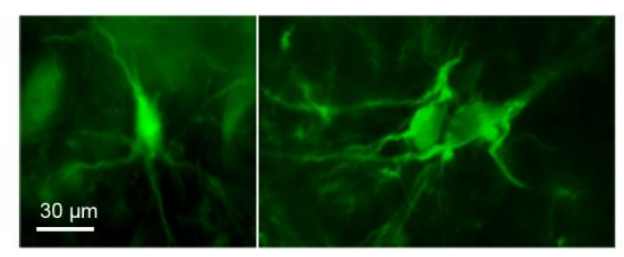

Fig. 3 


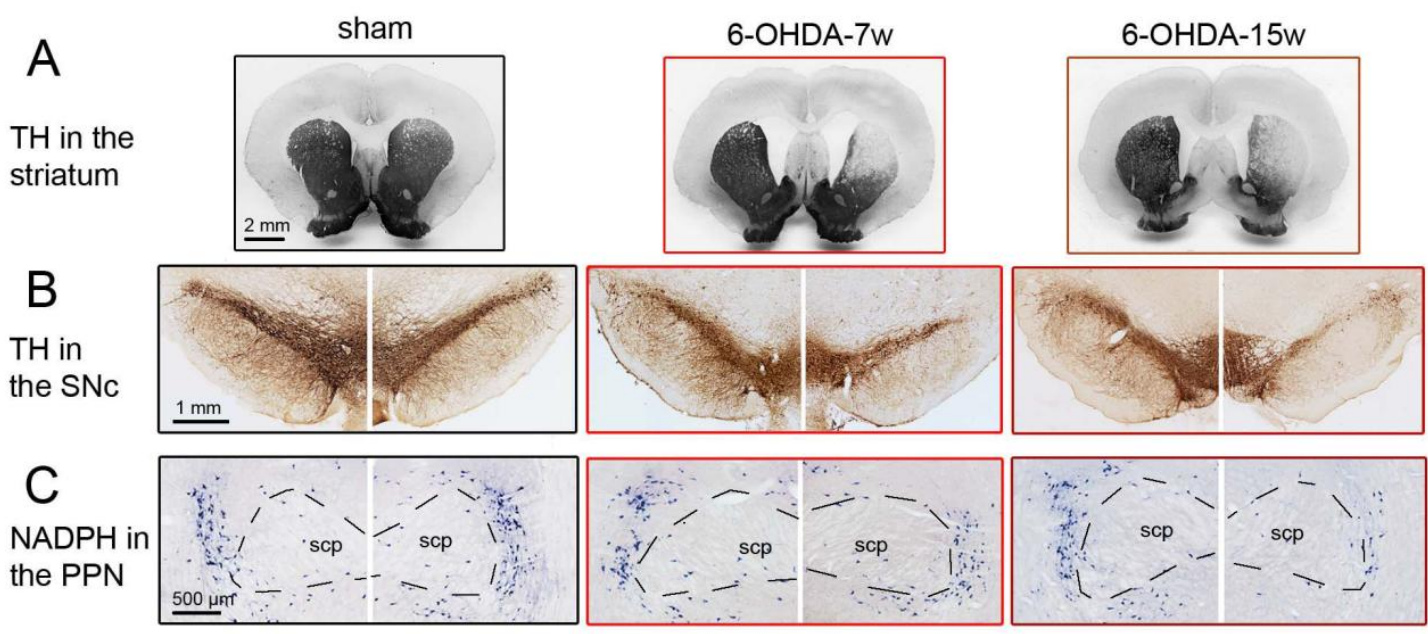

D
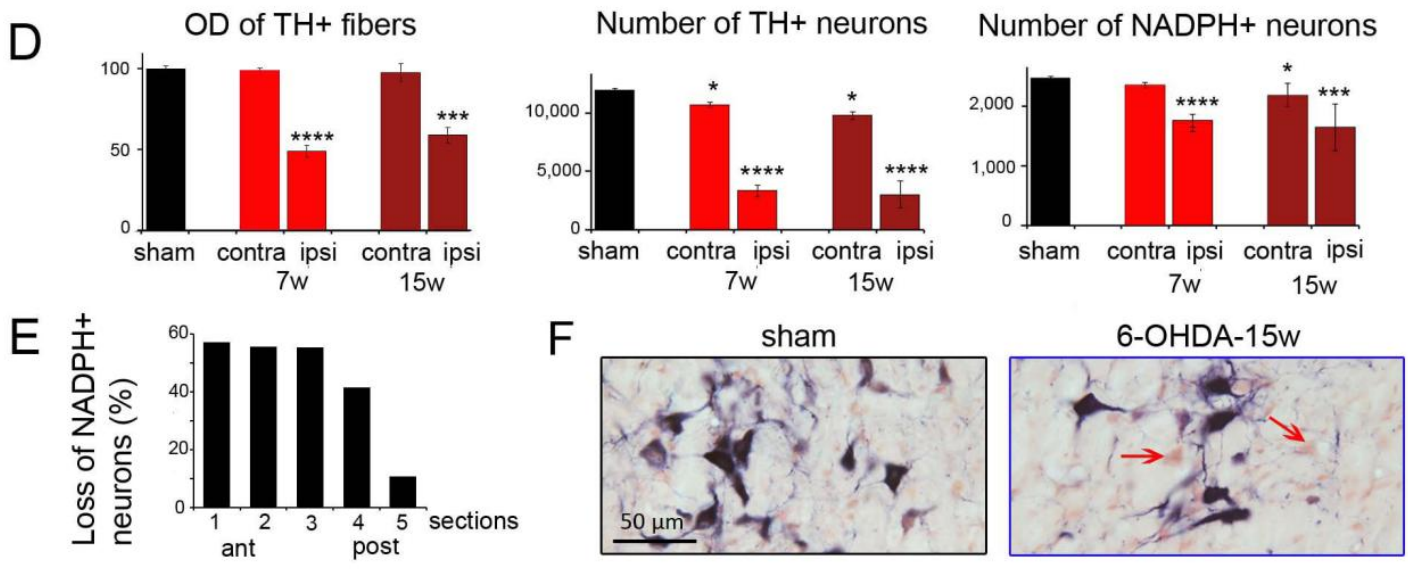

Fig. 4 

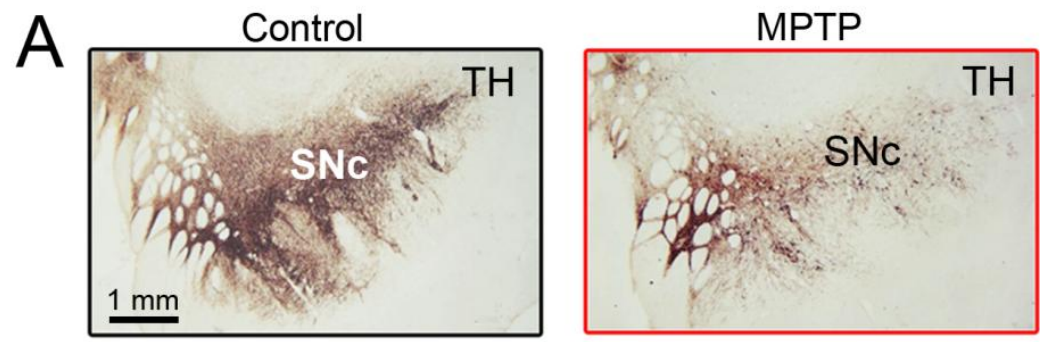

Total number of $\mathrm{TH}+$ neurons Total number of $\mathrm{NADPH}+$ neurons

B


Fig. 5 


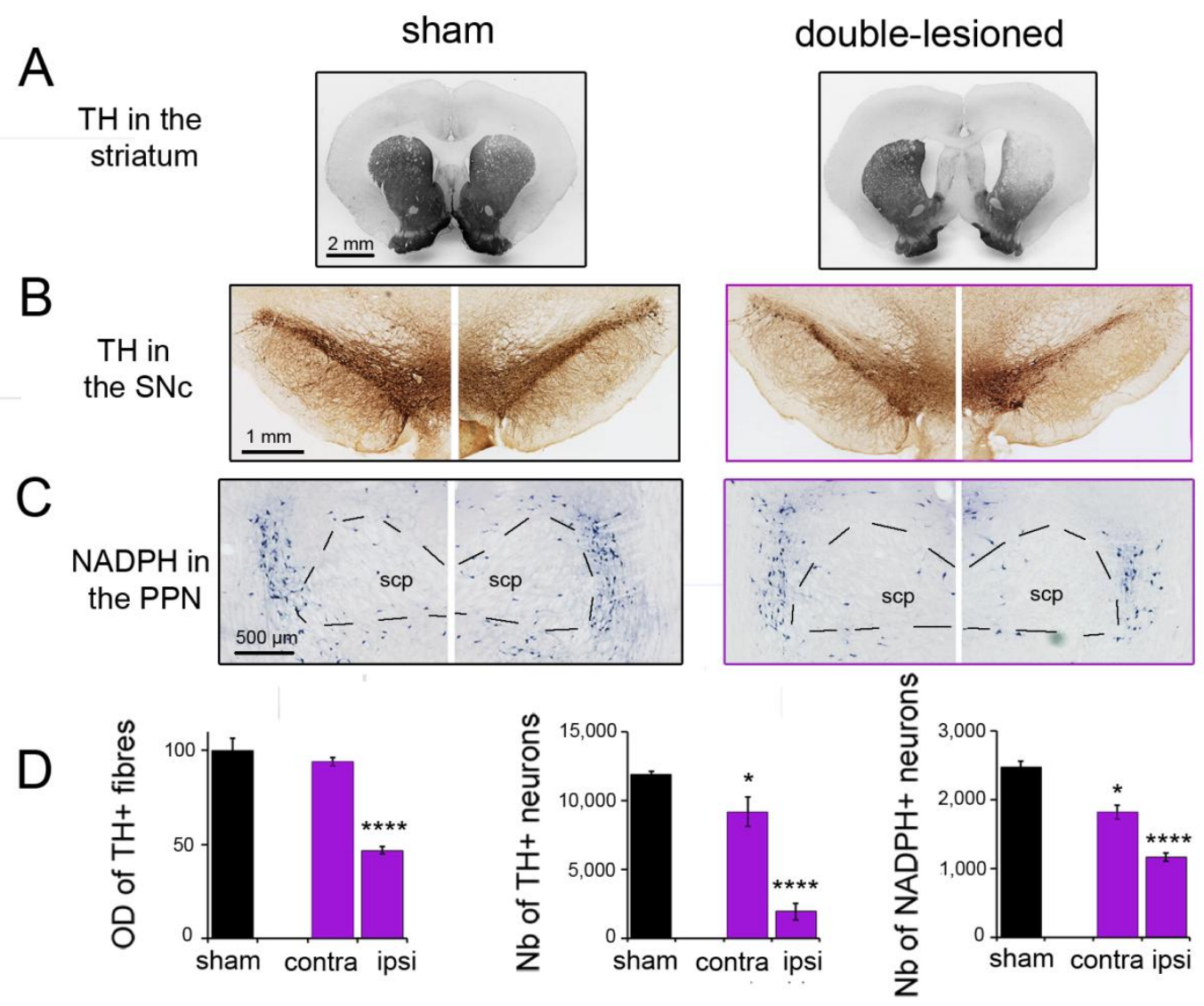

Fig. 6 

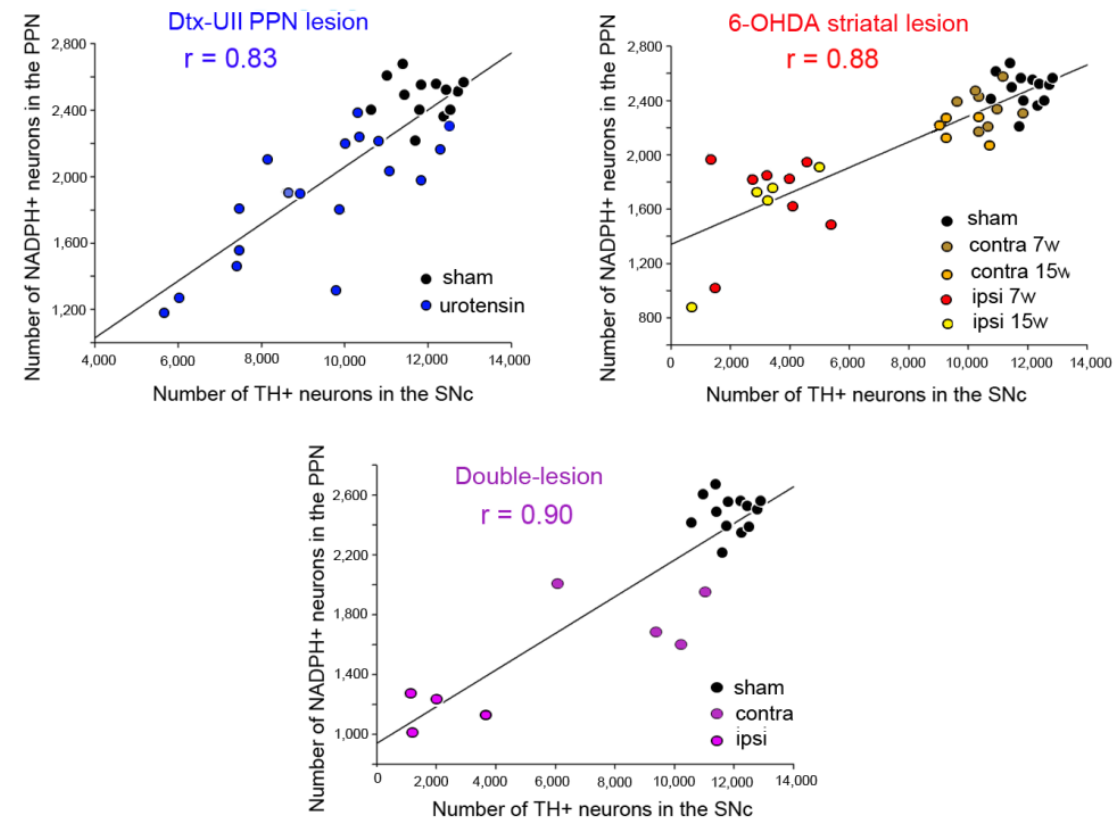

Fig. 7 
Table 1. Total number of NADPH+ in the PPN, of TH+ fibers in the striatum and of TH+ neurons in the SNc in the different experimental groups

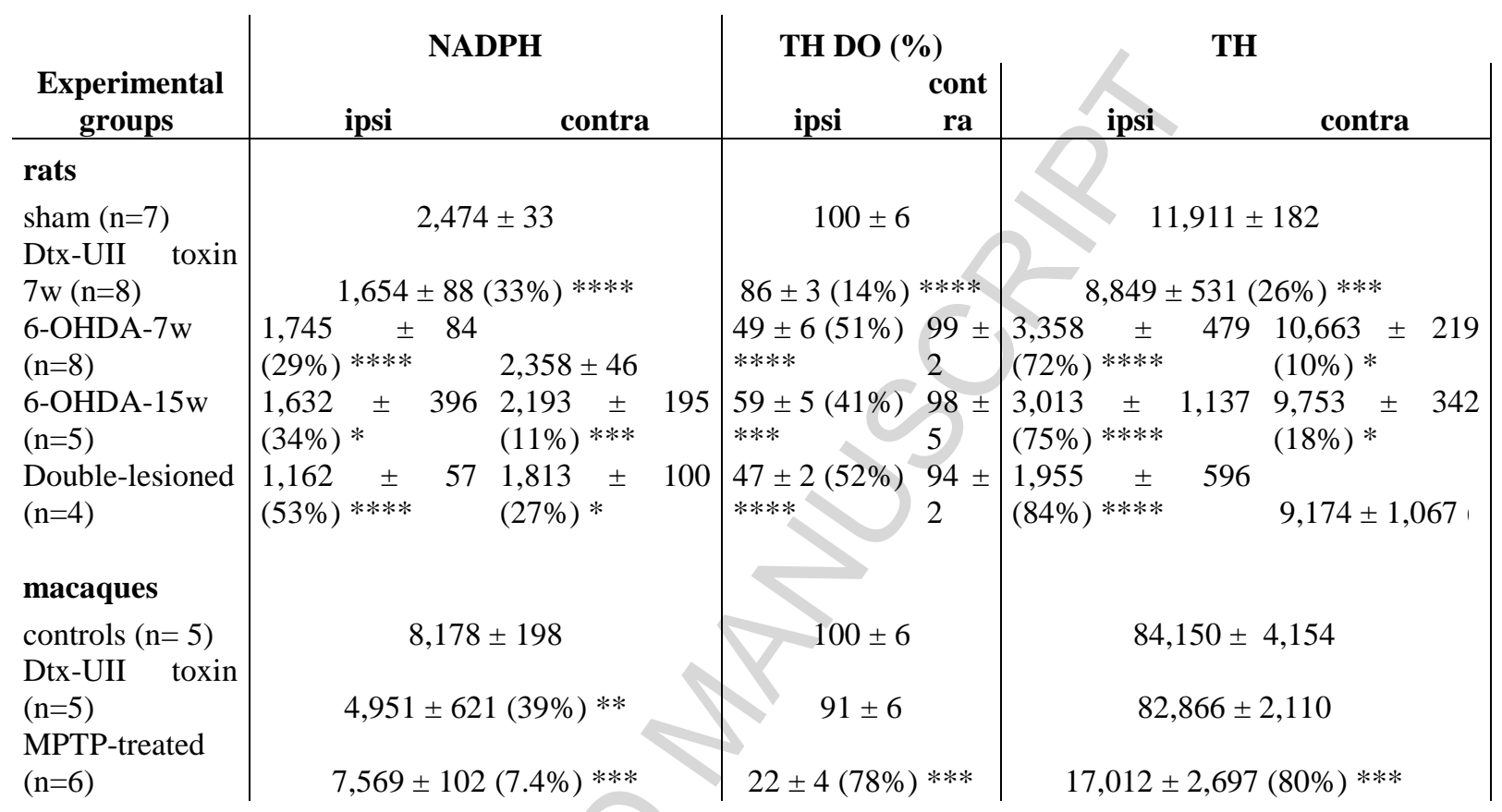

The number of neurons and fibers was given ipsi and contralaterally to the 6-OHDA lesion in rats. The extent of the lesions in the different experimental groups is expressed as a percentage of the respective control or sham value (in brackets). Mean \pm SEM. $* \mathrm{P}<0.05$, ** $\mathrm{P}<0.01$, *** $\mathrm{P}<0.005$, and $* * * * \mathrm{P}<0.001$, lesioned values versus sham or control values. 


\section{Highlights}

- We used lesion paradigms in normal and parkinsonian rats and monkeys

- Lesion of cholinergic pedunculopontine neurons affects dopaminergic neurons

- Dopaminergic lesion results in losses of cholinergic pedunculopontine neurons

- Double cholinergic and dopaminergic lesions aggravate neuronal losses

- Strong reciprocal interactions exist between dopaminergic and cholinergic systems 